\title{
Think Globally, Start Locally: Value-Based Breast Cancer Care for Newly Diagnosed Patients in A Safety-Net Medical Center
}

\author{
Annie Tang, MD², Shannon R Ugarte, MD', Amal L Khoury, MD, MPH², Bishal Gyawali, MD, PhD ${ }^{3}$, Anna Chiang ${ }^{4}$, \\ Nicole Lai ${ }^{4}$, Rohan E John ${ }^{5}$, Charles Bennett, MD, PhD, MPP ${ }^{6}$, Kevin Knopf, MD, MPH \\ ${ }^{1}$ Department of Medicine, Alameda Health System - Highland Hospital, Oakland, CA, USA, 2 Department of Surgery, University of California San \\ Francisco, East Bay - Highland Hospital, Oakland, CA, USA, ${ }^{3}$ Department of Medicine/Oncology, Queen's University, Kingston, Kingston, ON, Canada, \\ ${ }^{4}$ University of California, Berkeley, Berkeley, CA, USA, ${ }^{5}$ Department of Engineering, University of California, San Diego, San Diego, CA, USA, ${ }^{6}$ College \\ of Pharmacy, City of Hope Comprehensive Cancer Center, Duarte, CA, USA \& The University of South Carolina, Columbia, SC, USA \\ Keywords: cost effectiveness, breast cancer, safety net hospital, health services research
}

https://doi.org/10.53876/001c.31017

\section{International Journal of Cancer Care and Delivery}

Vol. 2, Issue 1, 2022

\section{Purpose}

We assessed the efficacy of a multidisciplinary, patient-focused approach emphasizing appropriate use of medical resources among a population of breast cancer patients at our safety-net hospital.

\section{Methods}

A multidisciplinary program coordinated and provided value-based care. Surgery, oncology, and navigation were physically co-located. Real time decisions were made by medical and surgical oncologists. Focused institution-specific protocols initiated in 2018, advised against four specific cancer resources that our team had determined as lower-value: imaging tests for indications not recommended in NCCN guidelines, inappropriate Oncotype Dx testing, radiation for patients $\geqslant 65$ years with stage I hormone-positive disease, and administration of pertuzumab and neratinib as adjuvant therapy in HER2+ breast cancer patients. Time to treatment and rates of use of these resources were monitored.

\section{Results}

Newly diagnosed breast cancer patients from 2015-2019 were compared to the pre-protocol era (2015-2017). Time from first breast clinic visit to oncology appointment decreased 39 days ( $60 \%$ decrease, median of 63.0 vs 22.5 days, $p<0.001$ ), no patients $\geqslant 65$ years with stage I hormone-positive breast cancer in 2018-2019 received radiation therapy, and rates of ordering of CT, PET, and bone scans for asymptomatic patients decreased by $80 \%$. Overall survival did not differ by cohort protocol category/treatment choices $(\mathrm{p}=0.69)$ Compared to the pre-protocol cohort, the post-protocol cohort did not have a significantly lower risk of death (Hazard Ratio 0.66 , 95\% Confidence Interval $0.08-5.38, p=0.69$ ). Overall breast cancer care cost decreased by $\$ 3,675,374$ between 2018 and 2019 versus 2015 to 2017.

\section{Conclusions}

After initiating a breast cancer program focused on reducing rates of use of four commonly excessively ordered breast cancer resources our team identified as lower-value, care at our safety-net hospital achieved high compliance with NCCN maging guidelines and also reduced use of a low-value diagnostic test, and low-value radiation and chemotherapy.

\section{INTRODUCTION}

The past decades have yielded progress in novel chemotherapy and genetic testing in breast cancer. These advancements, such as molecular profiling for risk assessment and targeted therapies, introduced an era of tailored treatment, leading to more complex clinical decision-making. ${ }^{1}$ This increase in advanced technology inevitably comes with a decrease in value-based cancer care. Valuebased care is central to recent Medicare-based cancer initiatives as these programs focus on costs and outcomes, generally at the system level. Treatment cost for one breast 
cancer patient in 2016 ranged from $\$ 71,909$ to $\$ 182,655$, 1.8-3.6 times breast cancer cost in 2009. ${ }^{2,3}$ Overall cancer costs are expected to rise from $\$ 183$ billion in 2015 to $\$ 246$ billion by $2030 .{ }^{4}$ As healthcare costs increase, valuebased care is essential and is becoming the focus of the next wave of cancer care. Rising prices increase price discrimination resulting in hospitals pursuing higher-revenue margins from privately-insured patients while Medicaid patients continue to go to hospitals with fewer resources. ${ }^{5}$ Using high-value diagnostic and treatment breast cancer care facilitates cost-effective care provision to lower-income cancer patients. Avoiding low-value cancer care by "choosing wisely" for breast cancer diagnostic and therapeutic care options (the cornerstone of the American Medical Association "Choosing Wisely" program developed for medical and surgical specialties) can help minimize breast cancer disparities.

Despite advances in breast cancer diagnostics and treatment, disparities in outcomes of patients in underserved communities persist. ${ }^{6}$ From 1989 to 2017, breast cancer morbidity rates dropped; yet racial mortality discrepancies persisted. ${ }^{7}$ Patients of lower socioeconomic status had worse clinical outcomes than patients of higher socioeconomic status. ${ }^{8}$ A recent study showed discordance with practicing care consistent with National Comprehensive Cancer Network (NCCN) care guidelines by race/ethnicity and socioeconomic status. ${ }^{9}$ While care coordination, shared patient registries, and patient navigators combat variations in the quality of cancer care, limited information exists on systematically implementing a prospective value-based approach to mitigate racial/ethnic cancer care disparities. ${ }^{10}$

Furthermore, two essential considerations for valuebased care should be noted. These include adhering to NCCN guideline practices and continually reviewing medical literature to critically avoid the use of unnecessary lowvalue diagnostics and treatment resources. Treatment goals for early-stage breast cancer are cure and decreasing local and systemic recurrence while maximizing quality-oflife (QoL). Several newer chemotherapy agents have been adopted whose use is of low value, Their FDA approvals were based on surrogate endpoints ${ }^{11}$ without clinical trials clearly identifying improved survival: Trastuzumab Emtansine (T-DM1) following neoadjuvant trastuzumab-based chemotherapy without a pathologic complete response (pCR), ${ }^{12}$ capecitabine following neoadjuvant chemotherapy without $\mathrm{pCR}$ in patients who are not triple negative, ${ }^{13}$ and adjuvant neratinib in human epidermal growth factor-2 positive (HER2+) patients. ${ }^{14}$ Aggressive use of imaging, such as positron-emission tomography (PET) and/or computed tomography (CT), is also of low-value, as use of these tests among women with early-stage breast cancer is unlikely to improve survival. While the use of genomic predictors, e.g. Oncotype Dx, is recommended in eligible patients, they are also of low value and are costly when compared to free validated online predictors that have shown similar prediction models to Oncotype Dx. ${ }^{15-17}$ Even adjuvant pertuzumab is of low-value as its use has not been shown to increase cure rates for women with HER2+ breast cancer despite its common usage. ${ }^{18}$ To provide a value-based cure for women with locally advanced breast cancer, careful analysis of commonly used practices with suboptimal evidence can help achieve value-based breast cancer care.

We sought to assess the efficacy of a multidisciplinary, patient-focused approach that minimized misuse, underuse, and overuse of societal healthcare resources while providing NCCN guideline-compliant care. We hypothesized that with a careful review of guidelines and literature, we could achieve NCCN-compliant breast cancer care by focusing on value-based care - preserving the stated goal of maximizing cure and improving processes of care. ${ }^{5}$

\section{METHODS}

We retrospectively analyzed breast cancer care at a single center between 2015 and 2019 after implementing our prospective value-based coordinated program. This study was approved by the Institutional Review Board at Highland Hospital, Oakland, California. Our institution is a safetynet county hospital in an urban setting serving a diverse low-socioeconomic community with a large minority population which can serve as a model for oncology care in lowand middle-income communities.

\section{INCLUSION AND EXCLUSION CRITERIA}

We included patients with newly-diagnosed breast cancer invasive ductal carcinoma, invasive lobular carcinoma, invasive mammary carcinoma, invasive mucinous carcinoma, and invasive papillary carcinoma. Patients with a diagnosis of ductal-carcinoma-in-situ, sarcoma, or metastasis from another organ system were excluded.

\section{PROTOCOL}

A community-based breast cancer multidisciplinary program was implemented in 2018 after a discussion with the oncologists and breast surgeons. We used a modified Donebanian approach ${ }^{19}$ combined with basic principles of cost-effectiveness analysis in our literature review. ${ }^{20}$ Our patient navigator, Full Time Equivalent $(\mathrm{FTE})=0.8$ with an annual salary of $\$ 68,000$ (provided by the American Cancer Society), provided rapid identification of patients, clinic coordination, social work assistance, and other patient needs. Weekly meetings among surgeons, oncologists, and the patient navigator were held to discuss patients, coordinate, and provide cost-effective efficient care. We co-located breast surgery, medical oncology, and navigator services in the same clinic. Joint-expedited decisions were made in real-time on baseline imaging, neoadjuvant/adjuvant chemotherapy/hormone therapy, and radiation in lieu of a formal tumor board for efficiency.

In 2018, value-based protocols for breast cancer management for newly-diagnosed patients were established. These were based on internal cost-effectiveness analysis ${ }^{19,20}$ study reviews where we reduced decisions to costminimization resulting in the following internal valuebased guidelines: 1) No patient with early-stage breast cancer would receive imaging outside of NCCN guide- 
Table 1. Cost Effective Diagnosis, Workup, and Treatment of Early-Stage Breast Cancer

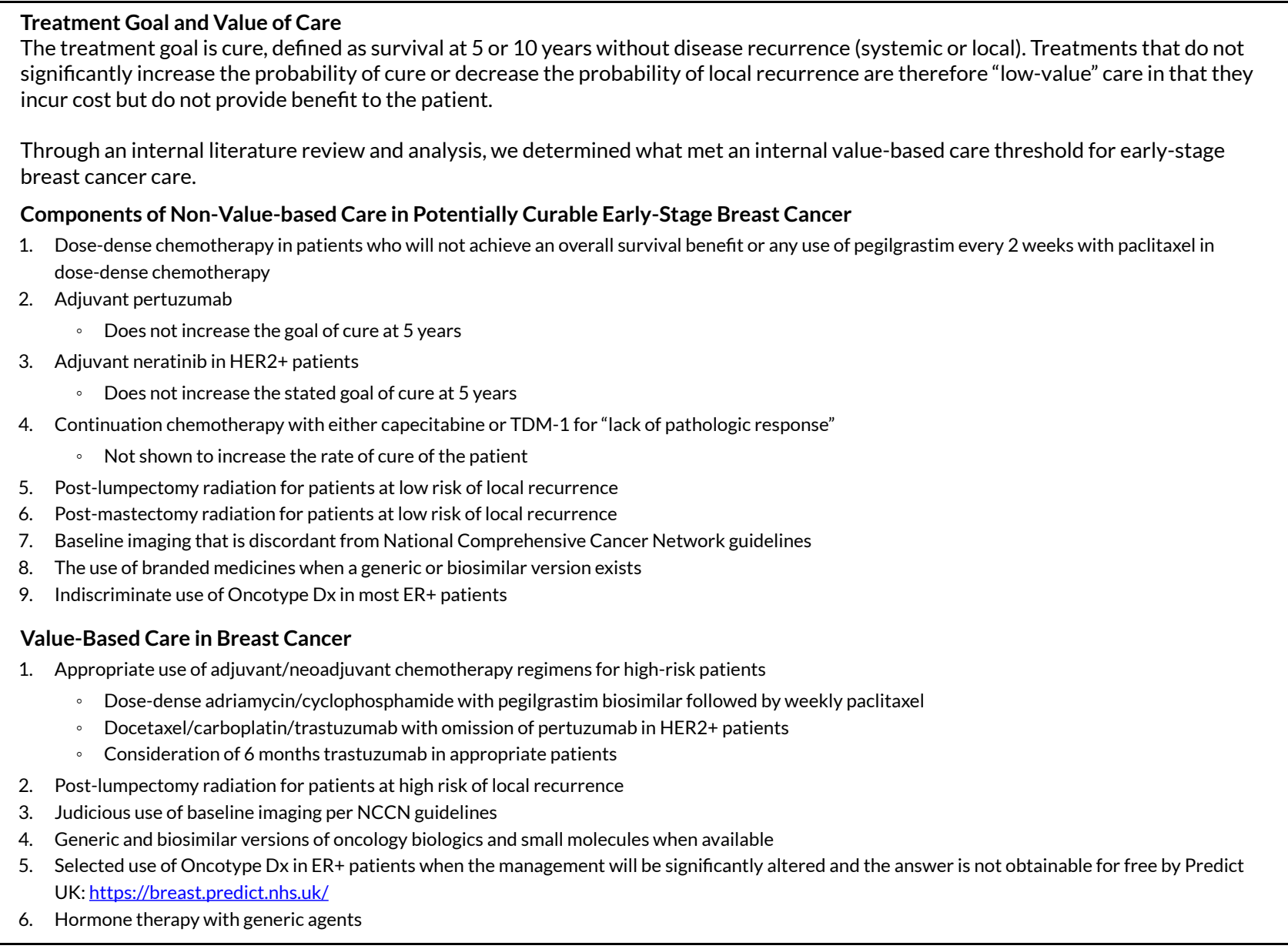

lines $^{21}$; 2) Oncotype Dx would be avoided in lieu of clinical judgment and use of open-access online nomograms ${ }^{15,16,22}$; 3) Avoidance of post-lumpectomy radiation in patients $\geqslant 65$ years with stage 1 hormone-receptor-positive $(\mathrm{HR}+)$ breast cancer ${ }^{23}$; 4) Omission of post-mastectomy radiation for women with 1-3 lymph nodes and tumors $<5 \mathrm{~cm}^{21} ; 5$ ) Avoidance of adjuvant pertuzumab based on a lack of significant improvement in cure rate 18,$24 ; 6$ ) Avoidance of T-DM1 in the adjuvant setting 25 ; 7) Avoidance of adjuvant neratinib in all HER2+ cancer. ${ }^{14}$ In NCCN imaging guidelines, ${ }^{21}$ for patients with T0-3, N1 breast cancer, CT chest, abdomen, and pelvis are indicated if symptoms, abnormal labs, or abnormal abdominal exams are present. Routine systemic staging is not indicated for early breast cancer in the absence of symptoms. For stage III or IV breast cancer, bone scans and PET scans are considered category $2 \mathrm{~b}$ (low-level evidence) for routine use without additional signs or symptoms. Imaging was critically assessed for utility and clinical suspicion (Table 1).

\section{DATA COLLECTION AND OUTCOMES}

Data collected included age at diagnosis, race, insurance, stage, and hormone status. Outcomes were time from breast surgery appointment to oncology appointment, time from oncology appointment to initial chemotherapy infusion, number of CT, PET, and bone scans ordered outside of
NCCN guidelines, radiation referrals for stage $1 \mathrm{HR}+$ breast cancer patients $\geqslant 65$ years, HER2+ patients who received pertuzumab or neratinib, and Oncotype Dx ordered.

Cost outcomes were derived from cost avoided. The estimate cost of diagnostics and treatment used were: $\$ 6,625$ for CT chest/abdomen/pelvis, ${ }^{26} \$ 5,300$ per PET scan, ${ }^{27}$ $\$ 750$ per nuclear bone scan, ${ }^{28} \$ 13,358$ for 6-week whole breast irradiation (WBI), ${ }^{29} \$ 164,807$ per course of neratinib, ${ }^{14} \$ 83,500$ annually of pertuzumab, 30 and $\$ 3,400$ per Oncotype Dx. ${ }^{31}$

The time required for physicians to review and discuss the literature was estimated to be 200 hours. Based upon physician salaries, the time was estimated to be equivalent to $\$ 30,000$. However, as the work was incorporated as regular duties by the navigator and physicians there were no incremental costs added.

\section{STATISTICAL METHODS}

Characteristics and outcomes were described by cohort year (2015-2019) and protocol category (post-protocol: 2018-2019 vs. pre-protocol: 2015-2017). Differences in categorical variables were compared using chi-square tests. Differences in continuous outcome variables were compared using Kruskal-Wallis tests. Graphs of the KaplanMeier survival estimates stratified by year and by cohort protocol category were produced for the death outcome, 
along with log-rank tests, and a Cox proportional hazards regression model was performed with cohort protocol category as the independent variable. The recurrence outcome, with competing risk event death, was summarized by year and cohort category. As the distributions of the time to clinic/chemotherapy variables were right-skewed, median regression results were reported for these outcomes. Hypothesis tests were two-sided and the significance threshold set to 0.05 . Statistical analyses were performed using SAS version 9.4

\section{RESULTS}

A total of 190 women with breast cancer were identified. The racial/ethnic distribution was 35.3\% African American, 26.3\% Asian, 25.3\% Hispanic, and 13.2\% Caucasian. Insurance status was $66.8 \%$ Medicaid, 23.2\% Medicare, and $10 \%$ uninsured. Most patients (75.2\%) presented at clinical stage I and II. Distributions of clinical stage and HER2 status differed by year. When pre- and post-protocol cohorts were compared, only the distribution of HER2 status differed significantly, with a higher percentage of HER2+ patients in the post-protocol cohort $(32.9 \%$ vs $11.6 \%$, p<0.01) (Table 2).

Median time from breast clinic to oncology clinic improved after protocol implementation by 39 days $(\beta=-39$, $\mathrm{SE}=7.6,95 \% \mathrm{CI}-54.1,-23.9, \mathrm{p}<0.01)$. This reduced time improved with each year (versus 2015, by 17 days in 2016, 21 days in 2017, 45 days in 2018 and 66 days in 2019). Median time from initial oncology appointment to chemotherapy did not differ when any of the years (2016-2019) were compared to 2015, or when the post-protocol cohort was compared to the pre-protocol cohort.

Imaging ordered outside of NCCN guidelines decreased significantly in the post-protocol period. Scans for asymptomatic patients decreased by $80 \%$ post-protocol. For stage $1 \mathrm{HR}+$ post-lumpectomy patients $\geqslant 65$ years, radiation therapy referrals decreased from $100 \%$ to $0 \%$; a total of six patients avoided radiation therapy.

Chemotherapy regimens varied by year and did not change after protocol initiation. Not all oncologists avoided pertuzumab. There was no decrease in use of adjuvant pertuzumab in HER2+ patients by year (from 2015 to 2019: $57 \%$, 25\%, 0\%, 77.8\%, 44.4\%, respectively). Post-protocol, $35 \%$ of the breast cancer patients who were HER2+ did not receive pertuzumab. Neither cohort received TDM-1 and neratinib. None of the HER2+ breast cancer patients $(n=18)$ received adjuvant neratinib.

Oncotype Dx testing varied by year (from 2015-2019: $0 \%, 17.2 \%, 38.7 \%, 10 \%, 0 \%$, respectively). Thirteen patients with early-stage $\mathrm{HR}+$, node-negative breast cancer in postprotocol cohort did not receive Oncotype Dx testing.

Survival rates differed significantly overall by year $(p=0.047)$ but did not differ by cohort protocol category $(\mathrm{p}=0.69)$ (Figure 1). Compared to the pre-protocol cohort, the post-protocol cohort did not have a significantly lower risk of death (Hazard Ratio 0.66, 95\% Confidence Interval 0.08-5.38, $\mathrm{p}=0.69$ ). Recurrence, with competing risk event

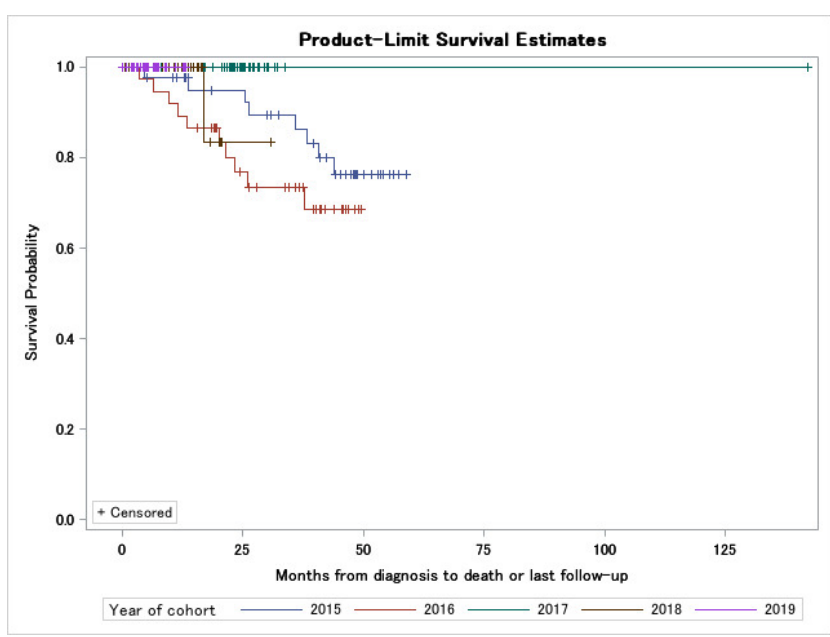

Figure 1a. Kaplan-Meier survival curves by year for patients diagnosed with breast cancer between 2015-2019.

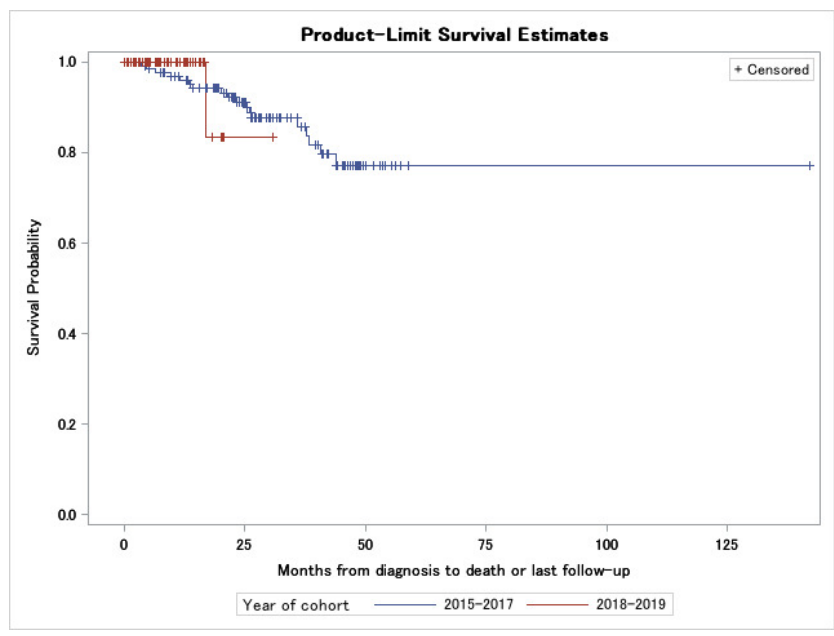

Figure 1b. Kaplan-Meier survival curves by cohort protocol category for patients diagnosed with breast cancer between 2015-2019.

death, did not differ significantly by year or by cohort protocol category.

When evaluating costs saved with four value-based treatment decisions (omission of neratinib, pertuzumab, oncotype testing, and radiation therapy in eligible patients), an estimated $\$ 3,675,374$ was saved in the post-protocol cohort, including costs of clinicians and the navigator to develop the treatment recommendations (Figure 2). Annual dollars spent on imaging referrals outside of NCCN guidelines decreased from $\$ 122,025$ in 2015 to $\$ 7,375$ in 2019.

\section{DISCUSSION}

To improve our use of value-based breast cancer care, we initiated a comprehensive program with protocols that resulted in coordinated care and reduced use of low-value diagnostic and treatment options for newly-diagnosed breast 
Table 2. Patient Characteristics by Year and Protocol Cohort

\begin{tabular}{|c|c|c|c|c|c|c|c|c|}
\hline Characteristic & & $\begin{array}{l}2015 \\
N=43\end{array}$ & $\begin{array}{l}2016 \\
N=38\end{array}$ & $\begin{array}{l}2017 \\
N=48\end{array}$ & $\begin{array}{l}2018 \\
N=25\end{array}$ & $\begin{array}{l}2019 \\
N=36\end{array}$ & $\begin{array}{l}\text { p-value } \\
\text { by Year }\end{array}$ & $\begin{array}{c}\text { p-value } \\
\text { by Cohort }\end{array}$ \\
\hline \multirow[t]{4}{*}{ Age, \% (n) } & & & & & & & 0.150 & 0.058 \\
\hline & $<50$ & $30.2 \%(13)$ & $23.7 \%(9)$ & $14.6 \%(7)$ & $32.0 \%(8)$ & $38.9 \%(14)$ & & \\
\hline & $50-64$ & $48.8 \%(21)$ & $47.4 \%(18)$ & $58.3 \%(28)$ & $28.0 \%(7)$ & $41.7 \%(15)$ & & \\
\hline & $\geq 65$ & $20.9 \%(9)$ & $28.9 \%(11)$ & $27.1 \%(13)$ & $40.0 \%(10)$ & $19.4 \%(7)$ & & \\
\hline \multirow[t]{5}{*}{ Race, \% (n) } & & & & & & & 0.103 & 0.202 \\
\hline & Asian & $32.6 \%(14)$ & $15.8 \%(6)$ & $16.7 \%(8)$ & $36.0 \%(9)$ & $36.1 \%(13)$ & & \\
\hline & Hispanic & $14.0 \%(6)$ & $28.9 \%(11)$ & $35.4 \%(17)$ & $12.0 \%(3)$ & $30.6 \%(11)$ & & \\
\hline & Caucasian & $9.3 \%(4)$ & $15.8 \%(6)$ & $18.8 \%(9)$ & $16.0 \%(4)$ & $5.6 \%(2)$ & & \\
\hline & African American & $44.2 \%(19)$ & $39.5 \%(15)$ & $29.2 \%(14)$ & $36.0 \%(9)$ & $27.8 \%(10)$ & & \\
\hline \multirow[t]{5}{*}{ Clinical stage, \% (n) } & & & & & & & 0.032 & 0.108 \\
\hline & 1 & $51.2 \%(22)$ & $39.5 \%(15)$ & $60.4 \%(29)$ & $40.0 \%(10)$ & $33.3 \%(12)$ & & \\
\hline & 2 & $30.2 \%(13)$ & $39.5 \%(15)$ & $33.3 \%(16)$ & $32.0 \%(8)$ & $61.1 \%(22)$ & & \\
\hline & 3 & $11.6 \%(5)$ & $7.9 \%(3)$ & $2.1 \%(1)$ & $8.0 \%(2)$ & $0.0 \%(0)$ & & \\
\hline & 4 & $7.0 \%(3)$ & $13.2 \%(5)$ & $4.2 \%(2)$ & $20.0 \%(5)$ & $5.6 \%(2)$ & & \\
\hline \multirow[t]{3}{*}{$E R, \%(n)$} & & & & & & & 0.151 & 0.415 \\
\hline & Neg & $32.6 \%(14)$ & $13.2 \%(5)$ & $16.7 \%(8)$ & $20.0 \%(5)$ & $30.6 \%(11)$ & & \\
\hline & Pos & $67.4 \%(29)$ & $86.8 \%(33)$ & $83.3 \%(40)$ & $80.0 \%(20)$ & $69.4 \%(25)$ & & \\
\hline \multirow[t]{3}{*}{$\mathrm{PR}, \%(\mathrm{n})$} & & & & & & & 0.906 & 0.692 \\
\hline & Neg & $41.9 \%(18)$ & $36.8 \%(14)$ & $35.4 \%$ (17) & $36.0 \%(9)$ & $44.4 \%(16)$ & & \\
\hline & Pos & $58.1 \%(25)$ & $63.2 \%(24)$ & $64.6 \%(31)$ & $64.0 \%(16)$ & $55.6 \%(20)$ & & \\
\hline \multirow[t]{3}{*}{ HER2, \% (n) } & & & & & & & 0.007 & $<0.001$ \\
\hline & Neg & $83.7 \%(36)$ & $89.5 \%$ (34) & $91.7 \%(44)$ & $64.0 \%(16)$ & $69.4 \%(25)$ & & \\
\hline & Pos & $16.3 \%(7)$ & $10.5 \%(4)$ & $8.3 \%(4)$ & $36.0 \%(9)$ & $30.6 \%(11)$ & & \\
\hline
\end{tabular}

ER= Estrogen Receptor; PR=Progesterone Receptor; HER2=Human Epidermal Growth Factor Receptor 2. 


\section{Costs Saved in the Post-Protocol (2018-2019) Cohort}

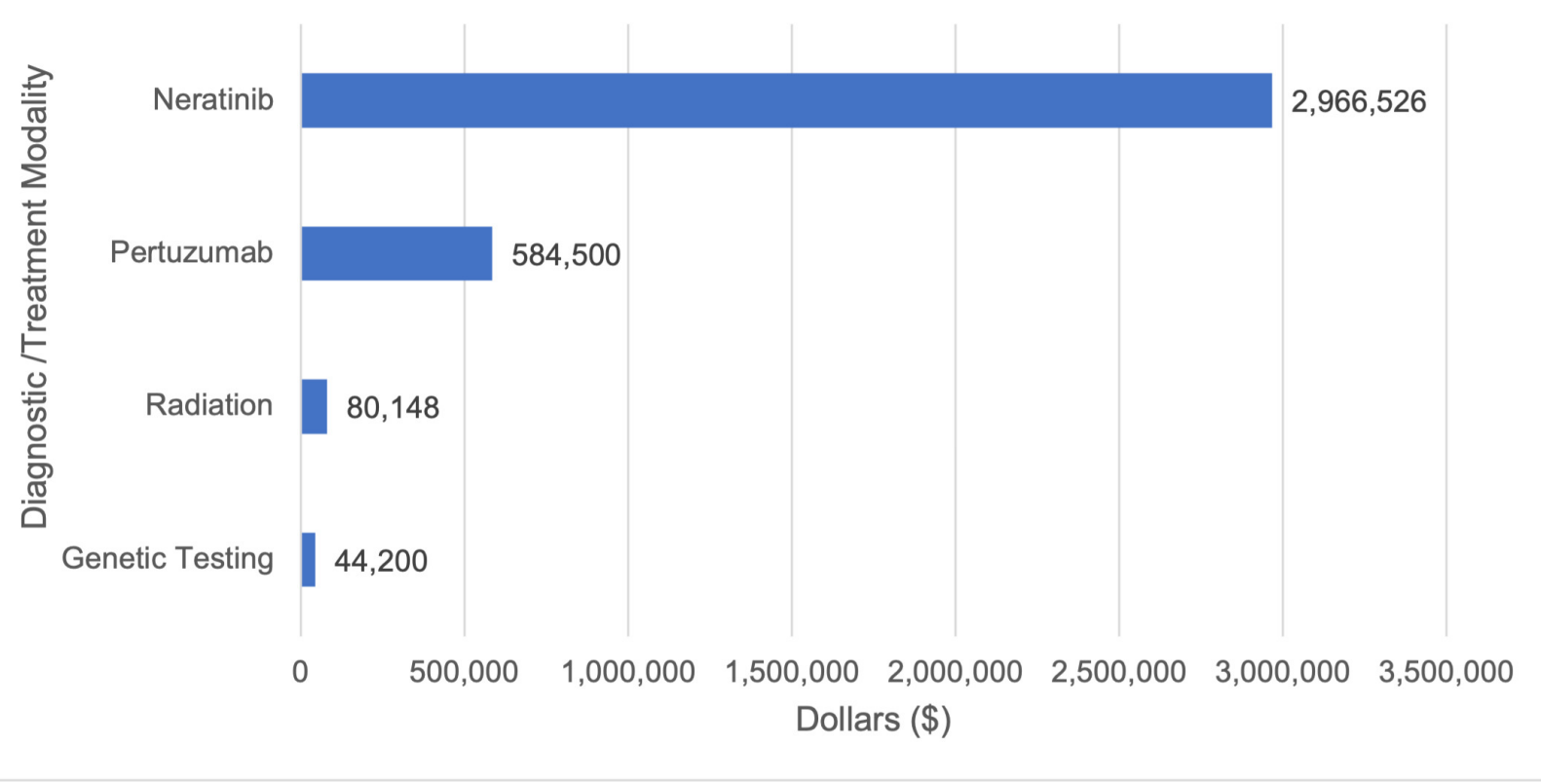

Figure 2. The total number of dollars saved from the omission of low-value diagnostics and treatment in the post-protocol (2018-2019) cohort.

cancer patients who received care at a safety-net medical system. After implementation in our hospital and outpatient clinics, we documented resource use with our focus on value-based care resulting in an estimated $\$ 3,675,374$ saved annually. Careful analysis of original data behind treatment recommendations can result in improved value and cost-savings to the health system (and also lower patient co-pays), while achieving similar short-term outcomes. Table 1 shows our value-based approach to diagnosis, workup, and treatment of newly diagnosed breast cancer in our health system. If applied to other similar safety-net-based breast cancer programs, this approach would save the health system a substantial amount of money without compromising patient outcomes.

Our value-based criteria for administering chemotherapy agents include therapy that improves overall survival with the goal of cure with no recurrence in 5-10 years - the treatment goal in adjuvant or neoadjuvant settings. Neratinib is a chemotherapy agent for HER2+ patients typically given after adjuvant trastuzumab. Neratinib costs $\$ 164,807$ per treatment course. ${ }^{14}$ Critical review of the 5 -year analysis of the ExteNet trial indicated that neratinib improves disease-free survival by 1.3 months, ${ }^{32}$ which may be analogous to early treatment of micro-metastatic disease but is not adjuvant in the true sense in that it does not improve cure rate. Compared to trastuzumab alone, trastuzumab plus neratinib is less cost-effective and does not improve overall survival. ${ }^{14}$ The APHINITY study showed a delay in recurrence, improvement in disease-free survival, and overall survival, but cure rate did not improve in the adjuvant setting. ${ }^{18,24}$ Overall survival may have improved because pertuzumab was initiated early, whereas the control arm had neither complete crossover nor access to pertuzumab when disease recurred. Pertuzumab, which costs $\$ 83,500$ annually, has been shown to be less cost-effective than trastuzumab alone. At our institution, instead of neratinib and pertuzumab, trastuzumab monotherapy is advocated for the treatment of HER2+ disease, with pertuzumab considered for neoadjuvant therapy. Our value-based care and associated cost savings are even better with the universal substitution of biosimilar trastuzumab for branded trastuzumab.

For patients with $\mathrm{HR}+$ node-negative breast cancer who were considered for chemotherapy, rates of ordering Oncotype Dx decreased in the post-protocol cohort. Previous studies showed that Oncotype Dx could provide prognostic information for patients, leading up to a $33.4 \%$ change in treatment recommendations as well as being a cost-effective method to reduce chemotherapy use. ${ }^{22,33-35}$ However, many of these studies relied on retrospective data that is hypothesis-generating rather than hypothesis-confirming. More cost-effective online tools for predicting patient risk for recurrence and need for chemotherapy exist, such as the NHS Predict UK ${ }^{16}$ and other nomograms. ${ }^{15}$ Oncotype Dx costs $\$ 3,400$ vs $\$ 0$ for online nomograms. ${ }^{31}$ At our institution, clinical judgment together with clinicopathologic criteria used in a nomogram or online tool (e.g. Predict UK) is encouraged to guide chemotherapy in select patients. Oncotype Dx is considered if uncertainty remains and its use is considered to possibly change management. Until a randomized study prospectively compares effectiveness between Oncotype Dx testing and online nomograms, the preferred value-based strategy at our institution is to use clinical judgement with a validated online prediction tool.

Another treatment that showed no improved survival is whole breast radiation in patients $\geqslant 65$ years with stage 1 
HR+ breast cancer. In the PRIME2 randomized controlled trial, whole breast radiation was compared to omission. ${ }^{23}$ After a 5-year follow-up, there was no difference in overall survival and a small decrease in 5-year recurrence from $4.1 \%$ in the whole breast radiation cohort to $1.3 \%$ in the radiation therapy omission cohort. ${ }^{23}$ We applied those results to our protocol, advocating for radiation therapy omission in eligible patients, and after protocol implementation in 2018, radiation therapy was omitted for all patients who met the criteria. Other studies have shown a range from $36.8 \%-52.8 \%$ radiation omission in the elderly. ${ }^{36,37}$ Widespread adoption of the de-escalation of breast radiation would save costs and decrease morbidity and inconvenience for patients who stand not to benefit in overall survival.

In addition to costs saved from treatment, decreased use of excess diagnostic tools resulted in cost reduction and was a strongly supported value-based recommendation. After incorporating NCCN imaging guidelines, CT scans were critically assessed for utility and clinical suspicion. In contrast to a study which showed increased discordance with NCCN guidelines by race, socioeconomic, and insurance status, ${ }^{9}$ we found an $80 \%$ decrease in CT imaging ordered outside of NCCN guidelines in 2018-2019 within our safetynet hospital that treats mostly racial/ethnic minorities of low socioeconomic status who are covered by Medicaid. Bone and PET scans ordered for asymptomatic patients without suspicious labs or imaging decreased to zero in 2018-2019, indicating that informed and updated valuebased guidelines implemented in our protocol helped reduce unnecessary imaging.

Our breast cancer program modifications resulted in decreased time from breast clinic to oncology clinic. Studies showed that delays in care, difficulties with access, and transportation are more prevalent in low socioeconomic communities. ${ }^{38-40}$ With surgery and oncology located in the same clinic, patients were likely able to reduce time away from work, transportation issues were lessened, and information was jointly obtained from surgical and medical oncologists at the same time which reduced confusion. Another value-based tool was a patient navigator. Patient navigators have been shown nationally to be cost-effective and can increase patient compliance and timely care. ${ }^{10,41,42}$ In communities where health knowledge is limited by language and cultural barriers, providing a consistent point of contact to ensure understanding and timely treatment is paramount. Lastly, regular meetings between the breast surgeons, oncologists, and patient navigators enabled timely concerns and decisions to be made more efficiently.
By decreasing difficulties of communication, focusing on care coordination, and reducing the number of patients lost to follow-up, we saw median time from breast surgery appointment to oncology appointment decrease from 63.0 days to 22.5 days. Currently, our patients are seen contemporaneously by a medical oncologist and a surgical oncologist whenever possible, which reduces the time to first oncology visit to zero days.

Our study has limitations. First, it is a retrospective review that compared two cohorts that were not matched, randomized, or controlled. Baseline characteristics also differed in HER2 status. Second, follow-up is skewed to longer follow-up for patients diagnosed in earlier years of the study period, leading to a lead-time bias. Moreover, the short follow-up period for patients diagnosed in the later years of the study, and limited sample size, preclude calculating accurate 5-year survival and recurrence rates. Longer-term follow-up is ongoing. Third, although protocols were initiated, clinical care was based on provider-patient shared decisions after informed discussions. The clinical choices of our three oncologists differed in varying degrees. For example, pertuzumab was not uniformly avoided. Depending on patient and provider preference, pertuzumab was sometimes given. Individual oncologists also differed regarding Oncotype Dx testing.

In conclusion, our study shows that after the implementation of value-based care options such as co-locating medical and surgical oncology clinics and providing access to value-based diagnosis and treatment protocols at our community breast cancer program in 2018-2019, we were able to significantly reduce costs with no apparent decrease in overall short-term survival and recurrence rates. The same set of rules and algorithms (Table 1) can be scaled across other safety-net cancer centers interested in providing value-based breast cancer care while preserving clinical outcomes. By reviewing current literature with scrutiny about important endpoints from patient and societal perspectives, and implementing value-based breast cancer protocols accordingly, we can provide better breast cancer care for our patients. In this regard, an American "safetynet" hospital that cares for poor and underserved patients can provide valuable information that can be evaluated for use in other safety-net medical systems seeking to maximize value-based care in an environment of economically constrained resources.

Submitted: September 20, 2021 PST, Accepted: December 18, 2021 PST 


\section{REFERENCES}

1. Harbeck N, Salem M, Nitz U, Gluz O, Liedtke C. Personalized treatment of early-stage breast cancer: present concepts and future directions. Cancer Treat Rev. 2010;36(8):584-594. doi:10.1016/j.ctrv.2010.04.0 $\underline{07}$

2. Campbell JD, Ramsey SD. The costs of treating breast cancer in the US: a synthesis of published evidence. Pharmacoeconomics. 2009;27(3):199-209. $\underline{\mathrm{d}}$ oi:10.2165/00019053-200927030-00003

3. Blumen H, Fitch K, Polkus V. Comparison of Treatment Costs for Breast Cancer, by Tumor Stage and Type of Service. Am Health Drug Benefits. 2016;9(1):23-32.

4. Mariotto AB, Enewold L, Zhao J, Zeruto CA, Yabroff KR. Medical Care Costs Associated with Cancer Survivorship in the United States. Cancer Epidemiol Biomarkers Prev. 2020;29(7):1304-1312. doi:10.1158/1 055-9965.EPI-19-1534

5. KaplanAlan, O’NeillDaniel. Hospital Price Discrimination Is Deepening Racial Health Inequity. NEJM Catalyst Innovations in Care Delivery. Published online December 21, 2020. Accessed March 1, 2021. $\underline{\mathrm{h}}$ ttps://catalyst.nejm.org/doi/full/10.1056/CAT.20.0593

6. Silber JH, Rosenbaum PR, Ross RN, et al.

Disparities in Breast Cancer Survival by

Socioeconomic Status Despite Medicare and Medicaid Insurance. Milbank Q. 2018;96(4):706-754. doi:10.111 1/1468-0009.12355

7. DeSantis CE, Ma J, Gaudet MM, et al. Breast cancer statistics, 2019. CA Cancer J Clin. 2019;69(6):438-451. doi:10.3322/caac.21583

8. Dreyer MS, Nattinger AB, McGinley EL, Pezzin LE. Socioeconomic status and breast cancer treatment. Breast Cancer Res Treat. 2018;167(1):1-8. doi:10.100 7/s10549-017-4490-3

9. Clair K, Chang J, Ziogas A, et al. Disparities by race, socioeconomic status, and insurance type in the receipt of NCCN guideline concordant care for select cancer types in California. JCO.

2020;38(15_suppl):7031-7031. doi:10.1200/JCO.202

0.38.15_suppl.7031

10. Battaglia TA, Freund KM, Haas JS, et al. Translating research into practice: Protocol for a community-engaged, stepped wedge randomized trial to reduce disparities in breast cancer treatment through a regional patient navigation collaborative. Contemp Clin Trials. 2020;93:106007. doi:10.1016/j.cc t.2020.106007
11. Prasad VK. Malignant: How Bad Policy and Bad Evidence Harm People with Cancer. Johns Hopkins University Press; 2020.

12. von Minckwitz G, Huang CS, Mano MS, et al. Trastuzumab Emtansine for Residual Invasive HER2-Positive Breast Cancer. N Engl J Med. 2019;380(7):617-628. doi:10.1056/NEJMoa1814017

13. Masuda N, Lee SJ, Ohtani S, et al. Adjuvant Capecitabine for Breast Cancer after Preoperative Chemotherapy. N Engl J Med. 2017;376(22):2147-2159. doi:10.1056/NEJMoa161264 $\underline{5}$

14. Schwartz NRM, Flanagan MR, Babigumira JB, Steuten LM, Roth JA. Cost-Effectiveness Analysis of Adjuvant Neratinib Following Trastuzumab in EarlyStage HER2-Positive Breast Cancer. J Manag Care Spec Pharm. 2019;25(10):1133-1139. doi:10.18553/jm cp.2019.25.10.1133

15. Orucevic A, Bell JL, McNabb AP, Heidel RE. Oncotype DX breast cancer recurrence score can be predicted with a novel nomogram using clinicopathologic data. Breast Cancer Res Treat. 2017;163(1):51-61. doi:10.1007/s10549-017-4170-3

16. Candido Dos Reis FJ, Wishart GC, Dicks EM, et al. An updated PREDICT breast cancer prognostication and treatment benefit prediction model with independent validation. Breast Cancer Res. 2017;19(1):58. doi:10.1186/s13058-017-0852-3

17. Orucevic A, Bell JL, King M, McNabb AP, Heidel RE. Nomogram update based on TAILORx clinical trial results - Oncotype DX breast cancer recurrence score can be predicted using clinicopathologic data. Breast. 2019;46:116-125. doi:10.1016/i.breast.2019.0 $\underline{5.006}$

18. Swain SM, Miles D, Kim SB, et al. Pertuzumab, trastuzumab, and docetaxel for HER2-positive metastatic breast cancer (CLEOPATRA): end-of-study results from a double-blind, randomised, placebocontrolled, phase 3 study. Lancet Oncol. 2020;21(4):519-530. doi:10.1016/S1470-2045(19)3086 3-0

19. Berwick D, Fox DM. "Evaluating the Quality of Medical Care”: Donabedian's Classic Article 50 Years Later. Milbank Q. 2016;94(2):237-241. doi:10.1111/14 68-0009.12189

20. Neumann PJ, Sanders GD, Russell LB, Siegal JE, Ganiats TG. Cost-Effectiveness in Health and Medicine. Second. Oxford University Press 2017; 2016. 
21. breast_blocks.pdf. Accessed September 10, 2020. $\underline{\mathrm{h}}$ ttps://www.nccn.org/professionals/physician_gls/pdf/ breast_blocks.pdf

22. Carlson JJ, Roth JA. The impact of the Oncotype Dx breast cancer assay in clinical practice: a systematic review and meta-analysis. Breast Cancer Res Treat. 2013;141(1):13-22. doi:10.1007/s10549-01 3-2666-z

23. Kunkler IH, Williams LJ, Jack WJL, Cameron DA, Dixon JM. Breast-conserving surgery with or without irradiation in women aged 65 years or older with early breast cancer (PRIME II): a randomised controlled trial. Lancet Oncol. 2015;16(3):266-273. do i:10.1016/S1470-2045(14)71221-5

24. Baselga J, Cortés J, Kim SB, et al. Pertuzumab plus trastuzumab plus docetaxel for metastatic breast cancer. N Engl J Med. 2012;366(2):109-119. doi:10.105 6/NEIMoa1113216

25. SABCS 2019: T-DM1 tradeoffs: not worth the costs and side effects in early stage breast cancer. Breast Cancer Action. Published December 12, 2019. Accessed September 10, 2020. https://bcaction.org/20 19/12/12/t-dm1-tradeoffs-not-worth-the-costs-and-s ide-effects-in-early-stage-breast-cancer/

26. Poslusny C. What is the cost of a CT scan in the U.S.? New Choice Health Blog. Published July 31, 2018. Accessed February 23, 2021. https://www.newc hoicehealth.com/ct-scan/cost

27. Whole Body PET Scan Cost and Procedure Comparison. NewChoiceHealth.com. Accessed February 23, 2021. https://www.newchoicehealth.co $\mathrm{m} /$ procedures/whole-body-pet-scan

28. Whole-Body Scans to Screen for Cancer | Choosing Wisely. Published November 18, 2015. Accessed February 23, 2021. https://www.choosingwi sely.org/patient-resources/whole-body-scans-to-scre en-for-cancer/

29. Greenup RA, Blitzblau RC, Houck KL, et al. Cost Implications of an Evidence-Based Approach to Radiation Treatment After Lumpectomy for EarlyStage Breast Cancer. J Oncol Pract. 2017;13(4):e283-e290. doi:10.1200/IOP.2016.016683

30. Beasley D. Roche combo of breast cancer drugs shows modest benefit. Reuters. https://www.reuters.c om/article/us-health-cancer-roche-idUSKBN18W1H A. Published June 5, 2017. Accessed February 23, 2021.
31. Genetic testing in women diagnosed with breast cancer decreases cost of care nationwide.

ScienceDaily. Accessed September 10, 2020. https://w ww.sciencedaily.com/releases/2019/04/19042408340 8.htm

32. Martin M, Holmes FA, Ejlertsen B, et al. Neratinib after trastuzumab-based adjuvant therapy in HER2-positive breast cancer (ExteNET): 5-year analysis of a randomised, double-blind, placebocontrolled, phase 3 trial. Lancet Oncol. 2017;18(12):1688-1700. doi:10.1016/S1470-2045(17)3 $\underline{0717-9}$

33. Buyse M, Loi S, van't Veer L, et al. Validation and clinical utility of a 70-gene prognostic signature for women with node-negative breast cancer. J Natl Cancer Inst. 2006;98(17):1183-1192. doi:10.1093/jnci/ djj329

34. Paik S, Shak S, Tang G, et al. A multigene assay to predict recurrence of tamoxifen-treated, nodenegative breast cancer. $N$ Engl J Med. 2004;351(27):2817-2826. doi:10.1056/NEJMoa041588

35. Tsoi DT, Inoue M, Kelly CM, Verma S, Pritchard KI. Cost-effectiveness analysis of recurrence scoreguided treatment using a 21-gene assay in early breast cancer. Oncologist. 2010;15(5):457-465. doi:1 0.1634/theoncologist.2009-0275

36. Gunn J, Lemini R, Partain K, et al. Trends in utilization of sentinel node biopsy and adjuvant radiation in women $\geqslant 70$. Breast $J$. 2020;26(7):1321-1329. doi:10.1111/tbj.13750

37. Pollock YG, Blackford AL, Jeter SC, et al. Adjuvant radiation use in older women with early-stage breast cancer at Johns Hopkins. Breast Cancer Res Treat. 2016;160(2):291-296. doi:10.1007/s10549-016-4005-7

38. Eley JW, Hill HA, Chen VW, et al. Racial differences in survival from breast cancer. Results of the National Cancer Institute Black/White Cancer Survival Study. JAMA. 1994;272(12):947-954. doi:10.1 001/jama.272.12.947

39. Dai D. Black residential segregation, disparities in spatial access to health care facilities, and late-stage breast cancer diagnosis in metropolitan Detroit. Health Place. 2010;16(5):1038-1052. doi:10.1016/j.hea lthplace.2010.06.012

40. Freeman HP. Patient navigation: a community based strategy to reduce cancer disparities. J Urban Health. 2006;83(2):139-141. doi:10.1007/s11524-00 $\underline{6-9030-0}$ 
41. Natale-Pereira A, Enard KR, Nevarez L, Jones LA. The role of patient navigators in eliminating health disparities. Cancer. 2011;117:3543-3552. doi:10.1002/ cncr.26264
42. Markossian TW, Calhoun EA. Are Breast Cancer Navigation Programs Cost-Effective? Evidence from the Chicago Cancer Navigation Project. Health Policy. 2011;99(1):52-59. doi:10.1016/j.healthpol.2010.07.00 $\underline{8}$ 